\section{Neue Myelinschicht durch altes Antiallergikum}

\author{
Eine Therapie mit dem Antiallergikum Clemastin könnte die Remyelinisierung \\ bei Multipler Sklerose ankurbeln. Darauf deutet eine kleine Studie bei Patien- \\ ten mit chronischer Optikusneuritis.
}

Patier atienten mit Optikusneuritis sind derzeit die Kandidaten der Wahl, wenn es um Tests mit potenziell remyelinisierenden Substanzen geht. Die Funktion des Sehnervs lässt sich hier über visuell evozierte Potenziale (VEP) gut messen. Je länger die Signale von der Retina in den visuellen Kortex benötigen, umso schlechter steht es um die Myelinschicht der betroffenen Sehnervenfasern. Vor allem die Potenzialspitze, die üblicherweise bei $100 \mathrm{~ms}$ liegt (P100), wird gerne als Hauptwert solcher VEPMessungen angegeben. Häufig wird sie gemessen, indem man den Patienten Schachbrettmuster zeigt, bei denen sich plötzlich der Kontrast umkehrt (weiß wird zu schwarz und umgekehrt). Bei MS-Patienten mit chronischer Optikusneuritis gelangen visuelle Signale der Kontrastumkehr verzögert ins Gehirn. Eine remyelinisierende Therapie sollte diese Latenz reduzieren. Offenbar scheint das nach einer Pilotstudie mit einem alten Antiallergikum zumindest ein bisschen zu klappen. Forscher um Ari Green von der Universität in San Francisco untersuchten 50 MS-Patienten (im Schnitt 40 Jahre alt und seit fünf Jahren an MS erkrankt), bei denen seit über einem Jahr eine Optikusneuritis bestand. Die P100-Latenz lag bei $127 \mathrm{~ms}$ und war damit deutlich erhöht, der EDSS-Wert erreichte knapp über 2 Punkte.

\section{P100-Latenz sinkt um 2 ms}

Das Team um Green wählte Clemastinfumarat, weil diese Substanz bei In-vitro-Screenings in der Lage war, die Oligodendrozytenreifung anzukurbeln. Wandeln sich mehr OligodendrozytenVorläuferzellen in reife Zellen um, sollte das die Myelinneubildung fördern. Von Vorteil war natürlich auch das langjährig gut bekannte Sicherheits- und Verträglichkeitsprofil von Clemastin. Eine Hälfte der Patienten erhielt zunächst zweimal täglich 5,4 mg Clemastinfumarat (entspricht $4 \mathrm{mg}$ Clemastin), die andere Placebo. Die Clemastin-Dosis lag etwa beim Vierfachen dessen, wie sie für eine antiallergische Behandlung verwendet wird. Nach 90 Tagen wurde gewechselt: Die Clemastin-Gruppe bekam nun für 60 Tage Placebo und umgekehrt.

Wie sich zeigte, ging der P100-Wert in der Gruppe, die mit Clemastin begann, innerhalb von ein bis zwei Monaten um knapp 2 ms zurück und blieb nach dem
Wechsel auf Placebo konstant. In der Gruppe, die mit Placebo anfing, blieb der Wert zunächst konstant und sank nach dem Wechsel auf Clemastin ebenfalls um rund 2 ms. Über alle Patienten gemittelt ging die P100-Latenz unter Clemastin im Vergleich zu Placebo um 1,9 ms signifikant zurück. Auch die Sehschärfe bei geringem Kontrast schien sich $\mathrm{zu}$ verbessern, wenngleich nicht signifikant.

\section{Fatigue nimmt zu}

Die Frage ist nun, wie relevant solche Verbesserungen beim P100-Wert tatsächlich sind, und ob sich die Latenz bei einer längeren Behandlung noch stärker senken lässt. Für die Forscher um Green ist das Ergebnis zumindest ein indirekter Hinweis darauf, dass sich die Oligodendrozytenvorläufer mit dem Wirkstoff auch in vivo bei MS-Patienten zur Differenzierung und Remyelinisierung anregen lassen - selbst bei chronischen Entzündungen. Ein Problem könnte jedoch die Sedierung sein, die gelegentlich unter dem Wirkstoff auftritt. So wurde in der Studie während der ClemastinBehandlung eine signifikante Zunahme der Fatigue beobachtet.

Thomas Müller, Springer Medizin

Green AJ et al. Positive double-blind randomized placebo-controlled crossover trial assessing the efficacy of clemastine fumarate for remyelination of chronic optic neuropathy in MS. Emerging Science Session. 68. AAN-Kongress, Vancouver, 15.-21.4.2016

\section{Nur etwas gesünder leben - weniger schwere Schlaganfälle}

Etwas weniger, essen, rauchen oder Alkohol trinken - für Frauen ab 50 kann sich das auszahlen: Ein geringfügig gesünderes Leben senkt nicht nur das Schlaganfallrisiko, sondern verbessert auch die Prognose nach einem Insult. haben mitunter eine große Wirkung: Es reicht vielleicht, das eine oder andere Laster etwas zu reduzieren und die Ernährung ein bisschen zu verbessern, um nicht nur das Schlaganfallrisiko zu senken, sondern einen Insult auch besser zu überstehen. Darauf deutet eine aktuelle Auswertung der Woman's Health Study.
Für Frauen besonders bedeutsam, da sie nach einem Schlaganfall oft stärker eingeschränkt sind als Männer.

\section{Schlaganfallrisiko halbiert}

Forscher um Dr. Pamela Rist von der Harvard Medical School in Boston haben Angaben von über 37.000 Frauen ausgewertet, die zum Beginn der Kohortenstudie im Mittel 54 Jahre alt waren. Bei allen waren die Lebensgewohnheiten erhoben und in einem Lebensstilindex subsummiert worden. Berücksichtigt wurden Rauchen, Alkoholkonsum, BMI, körperliche Bewegung sowie Ernährungsgewohnheiten. Bei maximal gesundem Lebensstil ließen sich 20 Punkte erzielen. 